\title{
The Farrell-Jones conjecture for graph products
}

\author{
GIOVANNI GANDINI \\ HENRIK RÜPING
}

\begin{abstract}
We show that the class of groups satisfying the $K$ - and $L$-theoretic Farrell-Jones conjecture is closed under taking graph products of groups.
\end{abstract}

18F25; 19A31, 19B28, 19G24

A group $G$ satisfies the $K$-theoretic Farrell-Jones conjecture with coefficients in additive categories if for any additive $G$-category $\mathcal{A}$ the assembly map (induced by the projection $E_{\mathcal{V C} y c} G \rightarrow \mathrm{pt}$ )

$$
\operatorname{asmb}_{n}^{G, \mathcal{A}}: H_{n}^{G}\left(E_{\mathcal{V C} y c} G ; \boldsymbol{K}_{\mathcal{A}}\right) \rightarrow H_{n}^{G}\left(\mathrm{pt} ; \boldsymbol{K}_{\mathcal{A}}\right)
$$

is an isomorphism. There is an analogous $L$-theoretic version of the Farrell-Jones conjecture where the $K$-theory of $\mathcal{A}$ is replaced by the $L$-theory of $\mathcal{A}$ with decoration $\langle-\infty\rangle$. We say that a group $G$ satisfies the FJC if for any finite group $F$ the group $G \nmid F$ satisfies the $K$ - and $L$-theoretic Farrell-Jones conjecture with coefficients in any additive category. The Farrell-Jones conjecture implies many famous conjectures, for example the Bass, the Borel, the Kaplansky and the Novikov conjectures; see Lück and Reich [9]. The Farrell-Jones conjecture for $K$-theory up to dimension one implies that the reduced projective class group $\widetilde{K}_{0}(R G)$ vanishes whenever $G$ is torsion-free and $R$ is a principal ideal domain. When $R$ is the ring of integers the vanishing of $\widetilde{K}_{0}(\mathbb{Z} G)$ implies Serre's conjecture, which claims that every group of type FP is of type FL.

Given a simplicial graph $\Gamma$ and a family of groups $\mathfrak{G}=\left\{G_{v} \mid v \in V \Gamma\right\}$, the graph product $\Gamma \mathfrak{G}$ is the quotient of free product $*_{v \in V \Gamma} G_{v}$ by the relations $\left[g_{u}, g_{v}\right]=1$ for all $g_{u} \in G_{u}, g_{v} \in G_{v}$ whenever $\{u, v\} \in E \Gamma$. Basic examples are given by right-angled Artin and right-angled Coxeter groups.

Let $\mathcal{C}$ be the class of groups satisfying the FJC. The purpose of this note is to prove the following closure property for the class $\mathcal{C}$.

Theorem 1 The class $\mathcal{C}$ is closed under taking graph products.

Since right-angled Artin and right-angled Coxeter groups are CAT(0)-groups they satisfy FJC by Bartels and Lück [2, Theorem B]. 
Acknowledgements We want to thank Yago Antolín and Sebastian Meinert for their comments. This work was financed by the Leibniz-Award of Professor Dr Wolfgang Lück granted by the Deutsche Forschungsgemeinschaft.

\section{Preliminaries}

Let us start by recalling some recent results of Bartels, Lück and Reich [3], Bartels, Farrell and Lück [1], Bartels and Lück [2] and Bartels, Lück, Reich and the second author [4].

Proposition 1.1 The class of groups $\mathcal{C}$ has the following properties:

(1) Word-hyperbolic groups belong to $\mathcal{C}$.

(2) CAT(0)-groups belong to $\mathcal{C}$.

(3) Virtually polycyclic groups belong to $\mathcal{C}$.

(4) The class $\mathcal{C}$ is closed under taking finite index overgroups.

(5) The class $\mathcal{C}$ is closed under taking subgroups.

(6) The class $\mathcal{C}$ is closed under taking directed colimits.

(7) The class $\mathcal{C}$ is closed under taking direct sums.

(8) Given any group homomorphism $f: G \rightarrow H$ and assume that $H \in \mathcal{C}$ and $f^{-1}(Z) \in \mathcal{C}$ for any torsion-free cyclic subgroup $Z$ of $H$; then, $G$ belongs to $\mathcal{C}$.

(9) The class $\mathcal{C}$ is closed under taking free products.

Proof The $K$-theoretic version without wreath products for hyperbolic groups was proved in [3, Main Theorem]. The $L$-theoretic version without wreath products for hyperbolic groups was proved in [2, Theorem B]. This generalizes to wreath products of hyperbolic groups with finite groups as explained in [4, Remark 6.4].

First note that a wreath product of a CAT(0) group and a finite group also acts geometrically on a CAT(0) space. Thus it suffices to consider the setting without wreath product which was proved in [2, Theorem B].

A wreath product of a virtually polycyclic group with a finite group is again virtually polycyclic. In [1, Theorem 0.1$]$ it was proved that virtually polycyclic groups satisfy Farrell-Jones.

By [1, Remark 0.5] the Farrell-Jones conjecture with finite wreath products passes to finite index overgroups. 
The remaining properties can be found in [1, Theorems 1.7-1.10] without wreath products. Note that if $H$ is a subgroup of $G$ then $H \imath F \leq G \imath F$, and hence it satisfies FJC.

We have for a direct system of groups $\left(G_{i}\right)_{i \in I}$ and a finite group $F$ that $\left.\left(\operatorname{colim}_{i \in I} G_{i}\right)\right\} F$ is isomorphic to $\operatorname{colim}_{i \in I}\left(G_{i} \succ F\right)$ and hence the $\mathbf{F J C}$ is also compatible with colimits.

Note that the wreath product of a direct sum by a finite group $F$ embeds in the direct sum of wreath products of the factors by $F$, hence we obtain that the FJC passes to finite direct sums. Hence by the previous point we have that $\mathcal{C}$ is closed under taking arbitrary direct sums.

Let $f: G \rightarrow H$ be a group homomorphism such that $H$ and $f^{-1}(Z)$ satisfy the FJC for any torsion-free cyclic subgroup $Z$ of $H$. Let $V$ be a virtually cyclic subgroup of $H$. Then it has a finite index torsion-free subgroup $Z$. Thus $f^{-1}(Z)$ has finite index in $f^{-1}(V)$. So $f^{-1}(V)$ also satisfies $\mathbf{F J C}$ by (4).

The same argument given by Kühl in [8, Lemma 3.16] for the fibered version of the Farrell-Jones conjecture applies to the FJC.

Consider the surjection $\pi: G_{1} * G_{2} \rightarrow G_{1} \times G_{2}$ and a torsion-free cyclic subgroup $C$ of $G_{1} \times G_{2}$. Note that by (7) $G_{1} \times G_{2}$ satisfies $\mathbf{F J C}$ and the subgroup $\pi^{-1}(C)$ acts on the Bass-Serre tree of $G_{1} * G_{2}$ with trivial edge stabilizers. Since for all $g \in G_{1} * G_{2}$, $G_{i}^{g} \cap \pi^{-1}(C)$ is either trivial or infinite cyclic, we have that $\pi^{-1}(C)$ is a free group and hence it satisfy $\mathbf{F J C}$ by (1).

In order to prove Theorem 1 we need to show that the fundamental groups of certain graphs of groups satisfy $\mathbf{F J C}$.

\section{Some special graphs of groups}

Basic facts about groups acting on trees and graphs of groups can be found in Serre [10]. Let us briefly recall how it is possible to recover a presentation for the fundamental group of a graph of groups $\mathcal{G}=\left(X, G_{\text {? }}, i_{*}: G_{*} \hookrightarrow G_{t(*)}\right)$. Pick a maximal tree $T \subset X$ and pick an orientation of each edge that is not in $T$. Let $L$ denote the set of those edges. Let $F(L)$ be the free group generated by $L$ and let $t_{e}$ be the generator corresponding to an edge $e \in L$. Then $\pi_{1}(\mathcal{G})$ is defined to be $*_{x \in V(X)} G_{X} * F(L)$ modulo the relations

$$
i_{e}(g)=i_{\bar{e}}(g) \quad \text { for } e \in T, g \in G_{e}, \quad t_{e} i_{e}(g) t_{e}^{-1}=i_{\bar{e}}(g) \text { for } e \notin T, g \in G_{e} .
$$


We have a canonical map $G_{v} \rightarrow \pi_{1}(\mathcal{G})$ for each vertex $v$. These turn out to be injective. Furthermore we can also get canonical maps $G_{e} \hookrightarrow \pi_{1}(\mathcal{G})$. We view $G_{e}$ as a subgroup of $G_{t(e)} \subset \pi_{1}(\mathcal{G})$ if either $e \in T$ or $e \notin T$ and $e$ has the chosen orientation.

Graphs of groups arise as quotients of groups acting on trees. The edge and vertex groups are given by the stabilizers. Every graph of groups arises uniquely in this way [10, Section I.5.3]. The acting group is isomorphic to the fundamental group of the induced graph of groups. This is the statement of the fundamental theorem for groups acting on trees [10, Theorem 13, Section I.5.4]. Thus any subgroup of $\pi_{1}(\mathcal{G})$ inherits a decomposition as a fundamental group of a graph of groups.

Definition 2.1 Let $\mathcal{G}$ be a countable graph of groups where every vertex group is either trivial or infinite cyclic. Since the edge groups inject into the vertex groups they are also either trivial or infinite cyclic. For every infinite stabilizer $G_{x}$ of a vertex $x$ pick a generator $z_{x}$. Similarly, for every edge $e$ with non trivial stabilizer $G_{e}$ let $z_{e}$ be the generator of $G_{e}$. For such an edge $e$ let $n_{e} \in \mathbb{Z}$ be defined by $i_{e}\left(z_{e}\right)=z_{t(e)}^{n_{e}}$. A closed edge loop $e_{1}, \ldots, e_{m}$ where each edge group is infinite cyclic is said to be balanced if

$$
\prod_{i=1}^{m} n_{e_{i}}=\prod_{i=1}^{m} n_{\bar{e}_{i}} .
$$

Note that picking different generators does not change balancedness since every minus sign appears twice. The graph of groups is said to be balanced if every closed edge loop with infinite cyclic edge stabilizers is balanced.

Example 2.2 The Baumslag-Solitar group $B S(m, n)=\left\langle a, b \mid b^{-1} a^{m} b=a^{n}\right\rangle$ is the fundamental group of a balanced graph of groups if and only if $m=n$.

Lemma 2.3 Let $\mathcal{G}$ be a finite balanced graph of groups with infinite cyclic vertex and edge groups. Then there is a central infinite cyclic subgroup $N \leq \pi_{1}(\mathcal{G})$ such that $\pi_{1}(\mathcal{G}) / N$ is isomorphic to the fundamental group of a finite graph of finite groups.

Proof For a vertex $v$ pick a generator $z_{v}$ of $G_{v} \leq \pi_{1}(\mathcal{G})$ and let $n:=\prod_{e \in E(\mathcal{G})} n_{e}$. Let $N:=\left\langle z_{v}^{n}\right\rangle \leq \pi_{1}(\mathcal{G})$ for some vertex $v$. Let us first show that $z_{v}^{n}$ is contained in every vertex group. Let $v^{\prime}$ be any vertex and let $e_{1}, \ldots, e_{m}$ be a path in $T$ from $v$ to $v^{\prime}$. Now let us use the relations corresponding to those edges. Note that from [10] we

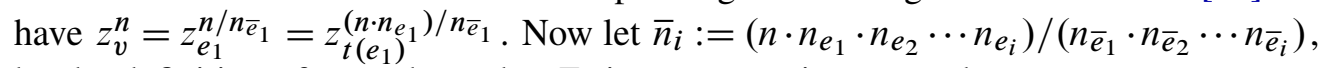
by the definition of $n$ we have that $\bar{n}_{i}$ is a nonzero integer and

$$
z_{v}^{n}=z_{t\left(e_{1}\right)}^{\bar{n}_{1}}=z_{t\left(e_{2}\right)}^{\bar{n}_{2}}=\cdots=z_{v^{\prime}}^{\bar{n}_{m}} .
$$

Thus $z_{v}^{n}$ is a power of $z_{v^{\prime}}$ and so both elements commute. 
Next we have to show that $z_{v}^{n}$ commutes with all elements of the form $t_{e}$ for some $e \in L$. Let $e_{1}, \ldots, e_{m}$ be a path in $T$ from $v$ to $t(e)$ and let $e_{1}^{\prime}, \ldots, e_{m^{\prime}}^{\prime}$ be a path in $T$ from $v$ to $t(\bar{e})$. Let us abbreviate

$$
\bar{n}_{i}:=\frac{n \cdot n_{e_{1}} n_{e_{2}} \cdots n_{e_{i}}}{n_{\bar{e}_{1}} n_{\bar{e}_{2}} \cdots n_{\bar{e}_{i}}}, \quad \bar{n}_{i}^{\prime}:=\frac{n \cdot n_{e_{1}^{\prime}} n_{e_{2}^{\prime}} \cdots n_{e_{i}^{\prime}}}{n_{\bar{e}_{1}^{\prime}} n_{\bar{e}_{2}^{\prime}} \cdots n_{\bar{e}_{i}^{\prime}}},
$$

as before. The path $e_{1}^{\prime}, \ldots, e_{m^{\prime}}^{\prime}, e, \bar{e}_{m}, \ldots, \bar{e}_{1}$ is closed and the balancedness condition gives

$$
\left(\prod_{i=1}^{m^{\prime}} n_{e_{i}^{\prime}}\right) \cdot n_{e} \cdot\left(\prod_{i=1}^{m} n_{\bar{e}_{i}}\right)=\left(\prod_{i=1}^{m^{\prime}} n_{\bar{e}_{i}^{\prime}}\right) \cdot n_{\bar{e}} \cdot\left(\prod_{i=1}^{m} n_{e_{i}}\right) .
$$

With the abbreviations from above this gives

$$
n_{e} \bar{n}_{m^{\prime}}^{\prime}=n_{\bar{e}} \bar{n}_{m} .
$$

Analogously to the previous case we get

$$
z_{t(e)}^{\bar{n}_{m}}=z_{v}^{n}=z_{t(\bar{e})}^{\bar{n}_{m^{\prime}}^{\prime}} .
$$

Let us conjugate the first part by $t_{e}$ and use the relation

$$
t_{e}\left(z_{t(e)}^{n_{e}}\right) t_{e}^{-1}=z_{t(\bar{e})}^{n_{\bar{e}}},
$$

which corresponds to $e$ to get

$$
t_{e} z_{v}^{n} t_{e}^{-1}=t_{e} z_{t(e)}^{\bar{n}_{m}} t_{e}^{-1}=\left(t_{e} z_{t(e)}^{n_{e}} t_{e}^{-1}\right)^{\frac{\bar{n}_{m}}{n_{e}}}=\left(z_{t(\bar{e})}^{n_{\bar{e}}}\right)^{\frac{\bar{n}_{m}}{n_{e}}}=z_{t(\bar{e})}^{\bar{n}_{m^{\prime}}^{\prime}}=z_{v}^{n} .
$$

In the first equality we used the left hand side of (2-2), in the third equality (2-3), the fourth equality uses (2-1) and the last equality uses the right-hand side of (2-2).

Thus $z_{v}^{n}$ commutes also with all generators of the form $t_{e}$ and hence $N$ is a central subgroup of $\pi_{1}(\mathcal{G})$. Furthermore the argument from above showed that $N$ is contained in any edge group.

Now let us pass to the actions on trees. Let $T^{\prime}$ be the universal cover of $\mathcal{G}$. Let us show that $N$ acts trivially. Let $e \in T^{\prime}$ be some edge. Its stabilizer is some conjugate of some edge group $G_{e} \subset \pi_{1}(\mathcal{G})$, say $g G_{e} g^{-1}$ for some $g \in \pi_{1}(\mathcal{G})$. Since $N$ is a central subgroup of $\pi_{1}(\mathcal{G})$ that is contained in each edge group $G_{e} \subset \pi_{1}(\mathcal{G})$ we have $N=g N g^{-1} \subset g G_{e} g^{-1}$. So the action factorizes as $\pi_{1}(\mathcal{G}) \rightarrow \pi_{1}(\mathcal{G}) / N \rightarrow \operatorname{Aut}\left(T^{\prime}\right)$. Especially note that the underlying graphs of $\pi_{1}(\mathcal{G}) \backslash T^{\prime}$ and $\left(\pi_{1}(\mathcal{G}) / N\right) \backslash T^{\prime}$ are the same. Thus $\pi_{1}(\mathcal{G}) / N$ can also be expressed as a fundamental group of a finite graph of groups. The vertex and edge groups are $G_{x} / N$. These are quotients of infinite cyclic groups by a nontrivial subgroup. Thus they are finite. 
Lemma 2.4 Let $\mathcal{G}$ be a balanced graph of groups. Then $\pi_{1}(\mathcal{G})$ satisfies the FJC.

Proof For the sake of simplicity we would like to define the fundamental group of a disconnected graph of groups as an assignment that assigns to any connected component the fundamental group of its graph of groups. First note that we can exhaust any graph by its finite subgraphs. Then its fundamental group will be the colimit of the fundamental group of the finite subgraphs of groups. So by Proposition 1.1(6) it suffices to consider a finite graph of groups with these properties.

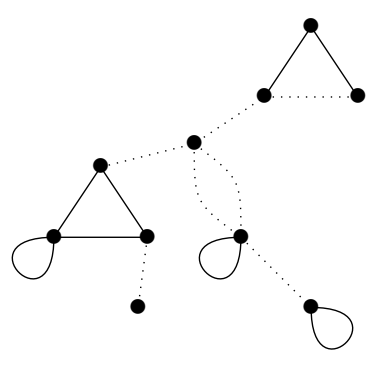

Figure 1: The dotted lines correspond to edges with trivial stabilizers and the lines correspond to edges with infinite cyclic stabilizers.

Next consider the subgraph consisting of all edges with nontrivial stabilizers and all vertices as illustrated in Figure 1. A connected component of a graph is a maximal, connected subgraph. Let us show that the fundamental group of each of its connected components satisfies the FJC. Note that an edge with nontrivial stabilizer can only connect vertices with nontrivial stabilizer. So the connected component of vertices with trivial stabilizers consists only of points and hence their fundamental group is trivial.

Let us now consider a connected component $\mathcal{C}$ of a vertex $v$ with nontrivial stabilizer. By Lemma 2.3 we find an infinite cyclic subgroup $N \leq \pi_{1}(\mathcal{C})$ such that $\pi_{1}(\mathcal{C}) / N$ is fundamental group of a finite graph of finite groups and hence virtually-free. So $\pi_{1}(\mathcal{C}) / N$ satisfies FJC by Proposition 1.1(1). Let us apply Proposition 1.1(8) to the map $f: \pi_{1}(\mathcal{C}) \rightarrow \pi_{1}(\mathcal{C}) / N$. Since the kernel of $f$ is infinite cyclic any preimage $f^{-1}(Z)$ of an infinite cyclic subgroup $Z$ of $\pi_{1}(\mathcal{C}) / N$ will satisfy the $\mathbf{F J C}$ by being virtually abelian.

Next we have to add edges to get the full graph. If we add an edge we either merge two connected components or add an edge to a single connect component. In the first case the fundamental group of the new component is the free product of the fundamental groups of the previous components. In the second case the new fundamental group is 
a free product of $\mathbb{Z}$ with the old fundamental group. In both cases the fundamental group of the new component satisfies the FJC by Proposition 1.1(9). This completes the proof.

The following criterion implies balancedness.

Lemma 2.5 Let $\mathcal{G}$ be a graph of groups where every vertex group is either trivial or infinite cyclic. Assume that for each edge $e$ with infinite cyclic stabilizer there exists a homomorphism $f_{e}: \pi_{1}(\mathcal{G}) \rightarrow \mathbb{Z}$ such that $\left.f_{e}\right|_{G_{e}}$ is nontrivial. Then $\mathcal{G}$ is balanced.

Proof Let us again use the notation of Definition 2.1. Recall that an edge $e^{\prime}$ gives a relation in the fundamental group. If the edge is contained in the chosen maximal tree, the relation will be $z_{t\left(\bar{e}^{\prime}\right)}^{n_{\bar{e}^{\prime}}}=z_{e^{\prime}}=z_{t\left(e^{\prime}\right)}^{n_{e^{\prime}}}$. Otherwise it will be $t_{e^{\prime}}^{-1} z_{t\left(\bar{e}^{\prime}\right)}^{n_{\bar{e}^{\prime}}} t_{e^{\prime}}=z_{e^{\prime}}=z_{t\left(e^{\prime}\right)}^{n_{e^{\prime}}}$. Since the target of $f_{e}$ is abelian we get in both cases $f_{e}\left(z_{t}\left(\bar{e}^{\prime}\right)\right)^{n_{\bar{e}^{\prime}}}=f_{e}\left(z_{t\left(e^{\prime}\right)}\right)^{n_{e^{\prime}}}$.

Let $e_{1}, \ldots, e_{m}$ be a loop of edges with infinite stabilizers, and let

$$
n:=\prod_{i=1}^{m} n_{e_{i}}, \quad \bar{n}:=\prod_{i=1}^{m} n_{\bar{e}_{i}} .
$$

So we get

$$
\begin{aligned}
f_{e_{m}}\left(z_{t\left(e_{m}\right)}\right)^{\bar{n}}=f_{e_{m}}\left(z_{t\left(\bar{e}_{1}\right)}\right)^{\bar{n}} & =f_{e_{m}}\left(z_{t\left(e_{1}\right)}\right)^{\bar{n} n_{e_{1}} / \bar{n}_{e_{1}}}=f_{e_{m}}\left(z_{t\left(\bar{e}_{2}\right)}\right)^{\bar{n} n_{e_{1}} / \bar{n}_{e_{1}}} \\
& =f_{e_{m}}\left(z_{t\left(e_{2}\right)}\right)^{\left(\bar{n} n_{e_{1}} n_{e_{2}}\right) /\left(\bar{n}_{e_{1}} \bar{n}_{e_{2}}\right)}=\cdots=f_{e_{m}}\left(z_{t\left(e_{m}\right)}\right)^{n} .
\end{aligned}
$$

Since $0 \neq f_{e_{m}}\left(z_{e_{m}}\right)=f_{e_{m}}\left(z_{t\left(e_{m}\right)}\right)^{n_{e m}}$, then $n=\bar{n}$. So the circle $e_{1}, \ldots, e_{m}$ is balanced and since the circle was chosen arbitrarily, the graph of groups is balanced.

Lemma 2.6 Given two groups $G_{1}, G_{2}$ with corresponding automorphisms $\varphi_{1}, \varphi_{2}$. Suppose that the FJC holds for $G_{1} \rtimes_{\varphi_{1}} \mathbb{Z}$ and for $G_{2} \rtimes_{\varphi_{2}} \mathbb{Z}$. Then it also holds for $\left(G_{1} \times G_{2}\right) \rtimes_{\varphi_{1} \times \varphi_{2}} \mathbb{Z}$.

Proof Projection to the first factor induces a homomorphism

$$
f:\left(G_{1} \times G_{2}\right) \rtimes_{\varphi_{1} \times \varphi_{2}} \mathbb{Z} \longrightarrow G_{1} \rtimes_{\varphi_{1}} \mathbb{Z} .
$$

We want to use Proposition 1.1(8), the target satisfies the Farrell-Jones conjecture with wreath products by assumption. The kernel is isomorphic to $G_{2}$ and hence it also satisfies the FJC by Proposition 1.1(5). Let $C$ be an infinite cyclic subgroup of $G_{1} \rtimes_{\varphi_{1}} \mathbb{Z}$ and let $(g, m)$ be a generator. Then $f^{-1}(C)$ is isomorphic to $G_{2} \rtimes_{\varphi_{2}^{m}} \mathbb{Z}$. For $m=0$ the preimage $f^{-1}(C)$ is just a direct product of two groups satisfying FJC. Otherwise $f^{-1}(C)$ is isomorphic to the subgroup $G_{2} \rtimes_{\varphi_{2}} m \mathbb{Z}$ and again by Proposition 1.1(5) it also satisfies the FJC. 
Lemma 2.7 Given two groups $G_{1}, G_{2}$ with automorphisms $\varphi_{1}, \varphi_{2}$. Suppose that the FJC holds for $G_{1} \rtimes_{\varphi_{1}} \mathbb{Z}$ and for $G_{2} \rtimes_{\varphi_{2}} \mathbb{Z}$. Then it also holds for $\left(G_{1} * G_{2}\right) \rtimes_{\varphi_{1} * \varphi_{2}} \mathbb{Z}$.

Proof We want to apply Proposition 1.1(8) to the canonical homomorphism

$$
f:\left(G_{1} * G_{2}\right) \rtimes_{\varphi_{1} * \varphi_{2}} \mathbb{Z} \longrightarrow\left(G_{1} \times G_{2}\right) \rtimes_{\varphi_{1} \times \varphi_{2}} \mathbb{Z} .
$$

The target satisfies the FJC by Lemma 2.6. The group $G_{1} * G_{2}$ acts on a tree $T$ with trivial edge stabilizers and vertex stabilizers conjugate to $G_{1}$ or $G_{2}$. We can identify the set of edges equivariantly with $G_{1} * G_{2}$ where the action is given by left multiplication. The set of vertices can be identified with $G_{1} * G_{2} / G_{1} \amalg G_{1} * G_{2} / G_{2}$ and the two endpoints of an edge are its cosets. We can extend the action to the group $\left(G_{1} * G_{2}\right) \rtimes_{\varphi_{1} * \varphi_{2}} \mathbb{Z}$ on the tree $T$ if we let the generator $t$ of $\mathbb{Z}$ act in the following way:

$$
\begin{aligned}
t \cdot g & :=\varphi_{1} * \varphi_{2}(g) & & \text { for an edge } g, \\
t \cdot g G_{i} & :=\varphi_{1} * \varphi_{2}\left(g G_{i}\right)=\varphi_{1} * \varphi_{2}(g) G_{i} & & \text { for a vertex } g G_{i} .
\end{aligned}
$$

The quotient of the tree $T$ by the group $\left(G_{1} * G_{2}\right) \rtimes_{\varphi_{1} * \varphi_{2}} \mathbb{Z}$ will be a segment since the quotient of $T$ by $G_{1} * G_{2}$ is a segment and $t$ does not swap the vertices. It is easy to see that this gives the following graph of groups decomposition for $\left(G_{1} * G_{2}\right) \rtimes_{\varphi_{1} * \varphi_{2}} \mathbb{Z}$ :

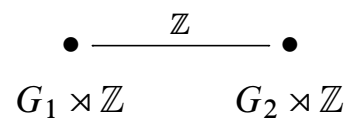

Now let $C$ be an infinite cyclic subgroup of $\left(G_{1} \times G_{2}\right) \rtimes_{\varphi_{1} \times \varphi_{2}} \mathbb{Z}$. The kernel of $f$ acts freely on $T$. So no nontrivial element from the kernel can stabilize some edge or some vertex. Restrict the group action to $f^{-1}(C)$, then $f$ maps injectively every stabilizer into $C$. So the group $f^{-1}(C)$ can be written as the fundamental group of a graph of groups $\mathcal{G}$ where every vertex group and every edge group is either infinite cyclic or trivial.

Any infinite edge group is subconjugate to $(1, \mathbb{Z}) \leq\left(G_{1} * G_{2}\right) \rtimes_{\varphi_{1} * \varphi_{2}} \mathbb{Z}$. So if we restrict the projection $\pi: \mathbb{Z} \leq\left(G_{1} * G_{2}\right) \rtimes_{\varphi_{1} * \varphi_{2}} \mathbb{Z} \rightarrow \mathbb{Z}$ to this edge group we get a nontrivial homomorphism. So $\mathcal{G}$ is balanced by Lemma 2.5. Hence $f^{-1}(Z)=\pi_{1}(\mathcal{G})$ satisfies the Farrell-Jones conjecture by Lemma 2.4. This completes the proof.

Let $S$ be a $\mathbb{Z}$-set and let $G$ be a group. We define $G_{S}:=\left(*_{s \in S} G\right) \rtimes \mathbb{Z}$ where $\mathbb{Z}$ acts on $*_{s \in S} G$ by $m \cdot g_{s}:=g_{m s}$. Here $g_{s}$ denotes the group element $g$ lying in the $s^{\text {th }}$ copy of $G$.

Lemma 2.8 For any $\mathbb{Z}$-set $S$ the group $G_{S}$ satisfies the FJC whenever $G$ does. 
Proof Any $\mathbb{Z}$-set is the disjoint union of its orbits. Furthermore, let $\bar{S}=\left\{S^{\prime} \subset S \mid\right.$ $S^{\prime}$ is a finite union of orbits $\}$. Then we have

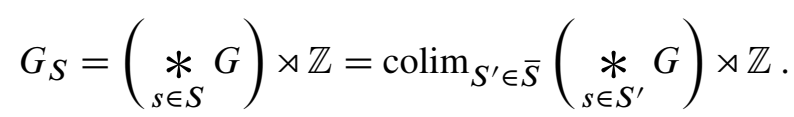

So by Proposition 1.1(6) it suffices to consider the case where $S$ has only finitely many orbits. By Lemma 2.7 it suffices to consider the case of a single orbit only.

If $S$ is of the form $\mathbb{Z} / m$ then the finite index subgroup $\left(*_{s \in \mathbb{Z} / m} G\right) \rtimes m \mathbb{Z}$ is isomorphic to a direct product $\left(*_{s \in \mathbb{Z} / m} G\right) \times \mathbb{Z}$ and so it satisfies the Farrell-Jones conjecture by Proposition 1.1(7) and Proposition 1.1(4). If $S$ is of the form $\mathbb{Z}$ we get $\left(*_{s \in \mathbb{Z}} G\right) \rtimes \mathbb{Z} \cong G * \mathbb{Z}$ and it also satisfies the FJC by Proposition 1.1(7).

Lemma 2.9 Let $\Gamma \mathfrak{G}$ be a graph product and $v \in V \Gamma$. Consider the graph $\Gamma^{\prime}$ obtained by removing $v$ from $\Gamma$ and let $\pi: \Gamma \mathfrak{G} \rightarrow \Gamma^{\prime} \mathfrak{G}$ be the canonical surjection. Then, for every cyclic subgroup $C$ its preimage $\pi^{-1}(C)$ is isomorphic to $\left(*_{\Gamma^{\prime} \mathfrak{G} / \Gamma^{\prime \prime G}} G_{v}\right) \rtimes C$ where $\Gamma^{\prime \prime}$ denotes the full subgraph of $\Gamma^{\prime}$ consisting of all neighbors of $v \in \Gamma$. The group $C$ acts on $*_{\Gamma^{\prime} \mathfrak{G} / \Gamma^{\prime \prime G}} G_{v}$ by permuting the free factors.

Proof By the argument given by Holt and Rees in the proof of [7, Theorem 4.1] the kernel of $\pi$ is isomorphic to $*_{\Gamma^{\prime} \mathfrak{G} / \Gamma^{\prime \prime} \mathfrak{G}} G_{v}$, where the isomorphism is given by $g_{h \Gamma^{\prime \prime} \mathfrak{G}} \mapsto g^{h}$. The preimage $\pi^{-1}(C)$ is isomorphic to $\left(*_{\Gamma^{\prime} \mathfrak{G} / \Gamma^{\prime \prime} \mathfrak{G}} G_{v}\right) \rtimes C$, where $C$ acts by conjugation on $*_{\Gamma^{\prime} \mathfrak{G} / \Gamma^{\prime \prime G}} G_{v}$. Moreover, using the explicit isomorphism from above we get that $C$ permutes the free factors.

Proof of Theorem 1 Let $v \in V \Gamma$ and let $\Gamma^{\prime}$ and $\Gamma^{\prime \prime}$ be defined as above. We apply Proposition 1.1(8) to the canonical surjection $\pi: \Gamma \mathfrak{G} \rightarrow \Gamma^{\prime} \mathfrak{G}$. The result follows immediately from an application of Lemmas 2.9 and 2.8 .

Remark Answering a long-standing open question ,Bestvina and Brady showed the existence of nonfinitely presented groups of type FP [5]. Given a nontrivial right-angled Artin group $G$ associated to a flag complex $L$ there is a canonical surjection $\phi: G \rightarrow \mathbb{Z}$ given by sending each generator to 1 . Bestvina and Brady proved that the finiteness properties of the kernel of $\phi$ are dictated by the connectivity property of $L$ and that the kernel of $\phi$ is of type FP if and only if is of type FL. Now consider a graph product $\Gamma \mathfrak{G}$ such that every vertex group $G_{v}$ surjects onto $\mathbb{Z}$. These maps induce a surjection $\phi_{V}: \Gamma \mathfrak{G} \rightarrow \mathbb{Z}$ and it would be interesting to study the finiteness property of the kernel of $\phi_{V}$. Note that whenever the copies of $G_{v}$ satisfy the FJC it follows from Theorem 1 and Proposition 1.1(5) that so does $\operatorname{ker} \phi_{V}$. Hence $\operatorname{ker} \phi_{V}$ cannot solve in negative Serre's conjecture. However, there are many indicable groups for which the FJC is unknown, some examples are given by nonsolvable Baumslag-Solitar groups (see Farrell and $\mathrm{Wu}[6]$ for the solvable ones). 


\section{References}

[1] A Bartels, F T Farrell, W Lück, The Farrell-Jones conjecture for cocompact lattices in virtually connected Lie groups arXiv:1101.0469

[2] A Bartels, W Lück, The Borel conjecture for hyperbolic and CAT(0)-groups, Ann. of Math. 175 (2012) 631-689 MR2993750

[3] A Bartels, W Lück, H Reich, The K-theoretic Farrell-Jones conjecture for hyperbolic groups, Invent. Math. 172 (2008) 29-70 MR2385666

[4] A Bartels, W Lück, H Reich, H Rüping, $K$ - and L-theory of group rings over $\mathrm{GL}_{n}(Z)$, to appear in Publ. Math. IHES arXiv:1204.2418

[5] M Bestvina, N Brady, Morse theory and finiteness properties of groups, Invent. Math. 129 (1997) 445-470 MR1465330

[6] T Farrell, X Wu, Farrell-Jones conjecture for the solvable Baumslag-Solitar groups arXiv: 1304.4779

[7] D F Holt, S Rees, Generalising some results about right-angled Artin groups to graph products of groups, J. Algebra 371 (2012) 94-104 MR2975389

[8] P Kühl, Isomorphismusvermutungen und 3-Mannigfaltigkeiten arXiv:0907.0729

[9] W Lück, H Reich, The Baum-Connes and the Farrell-Jones conjectures in $K$ - and $L$-theory, from: "Handbook of $K$-theory, Vol. 1, 2", (E M Friedlander, D R Grayson, editors), Springer, Berlin (2005) 703-842 MR2181833

[10] J-P Serre, Trees, Springer Monographs in Mathematics 9, Springer, Berlin (2003) MR1954121

Institut for Matematiske Fag, Københavns Universitet Universitetsparken 5, DK-2100 København, Denmark

Mathematisches Institut, Rheinische Wilhelms-Universität Bonn

Endenicher Allee 60, D-53115 Bonn, Germany

ggandini@math.ku.dk, henrik.rueping@hcm.uni-bonn.de

http://www.math.ku.dk/ zjb179,

http://www.math.uni-bonn.de/people/rueping

Received: 7 December 2012 